\title{
Enhanced thermal and mechanical properties of epoxy composites by mixing thermotropic liquid crystalline epoxy grafted graphene oxide
}

\author{
B. Qi, S. R. Lu*, X. E. Xiao, L. L. Pan, F. Z. Tan, J. H. Yu
}

Key Laboratory of New Processing Technology for Nonferrous Metals and Materials, Ministry of Education, School of Material Science and Engineering, Guilin University of Technology, 541004 Guilin, China

\begin{abstract}
Graphene oxide (GO) sheets were chemically grafted with thermotropic liquid crystalline epoxy (TLCP). Then we fabricated composites using TLCP-g-GO as reinforcing filler. The mechanical properties and thermal properties of composites were systematically investigated. It is found that the thermal and mechanical properties of the composites are enhanced effectively by the addition of fillers. For instance, the composites containing $1.0 \mathrm{wt} \%$ of TLCP-g-GO present impact strength of $51.43 \mathrm{~kJ} / \mathrm{m}^{2}$, the tensile strength of composites increase from 55.43 to $80.85 \mathrm{MPa}$, the flexural modulus of the composites increase by more than $48 \%$. Furthermore, the incorporation of fillers is effective to improve the glass transition temperature and thermal stability of the composites. Therefore, the presence of the TLCP-g-GO in the epoxy matrix could make epoxy not only stronger but also tougher.
\end{abstract}

Keywords: nanocomposites, thermotropic liquid crystalline epoxy, graphene oxide, mechanical properties, thermal properties

\section{Introduction}

Epoxy resins are presently important organic matrices in composite industry. They are frequently used in demanding applications due to their excellent mechanical properties, thermal stability and chemical resistance. Furthermore, they also have good resistance to moisture, solvents and chemical attacks $[1,2]$. However, their brittleness, poor resistance to crack propagation and poor wear resistance $[3,4]$ limit their applications. Numerous studies have been conducted to explore the toughness of epoxy composites $[5,6]$. Addition of thermoplastic polymers can improve fracture toughness, but it is always at the expense of the glass transition temperature $\left(T_{\mathrm{g}}\right)$ and modulus or high cost [7]. Inorganic additives, such as silica and alumina have been used to increase the toughness of epoxies without sacrificing their basic properties, but the presence of numerous inor- ganic particles increase the viscosity leading to poor dispersion and processing difficulty [8-10]. In recent years, graphene oxide (GO) has attracted significant interest for the preparation of polymer composites due to its excellent mechanical [11], electrical and thermal properties, simple preparation technique and low cost [12]. GO can be regarded as graphene sheets derivatized with carboxylic groups at the edges and phenol, hydroxyl and epoxide groups on the basal planes [13-15]. These abundant oxygen-containing functional groups enable graphene to be further modified by chemical reactions, and thus many different functional groups can be introduced onto the graphene sheets. Accordingly, we can easily to prepare a variety of excellent functional composites.

Liquid crystalline epoxies (LCE) form a unique class of thermosetting materials, which combine the

\footnotetext{
${ }^{*}$ Corresponding author, e-mail: lushaor@163.com

(C) BME-PT
} 
properties of both liquid crystal and conventional thermosets, as shown by their anisotropic mechanical properties, excellent thermal stability and chemical resistance [16-18].Curing of low molecular weight, rigid rod, and multifunctional monomers result in the retention of a liquid crystalline phase by the three dimensional crosslinking networks [19, 20]. Hence, it can be used to improve toughness of composites. Unlike other additive such as rubber particles, the presence of liquid crystalline domains will not sacrifice $T_{\mathrm{g}}$ or modulus of the material. These outstanding properties make LCE as good candidates for a wide range of potential applications, such as optical switches, electronic packaging, and matrices for high performance composites [21-23]. In this report, we firstly prepared GO by a modified Hummers method, then the GO was grafted by thermotropic liquid crystalline epoxy (TLCP-g-GO). The GO and grafted GO were characterized by Fourier-transform infrared spectra (FT-IR), wide angle $\mathrm{X}$-ray diffraction (WAXD) and so on. Meanwhile, the epoxy composites were prepared by casting with the addition of $0.5,1.0,1.5,2.0$ and $3.0 \mathrm{wt} \%$ TLCPg-GO. Finally, we studied the effect of the TLCP-gGO on mechanical and thermal properties of the epoxy composites.

\section{Experimental section}

\subsection{Materials}

Nature flake graphite (325 mesh, 99\%) was provided by Hengrui Graphite Co., Ltd. (Qingdao, China). Phydroxybenzoic acid and paraphthaloyl chloride were obtained from Xiya Chemical Reagent Company (Chengdu, China). Thionyl chloride $\left(\mathrm{SOCl}_{2}\right)$ and glycidol were purchased from Aladdin Chemistry Co., Ltd. (Shanghai, China). $\mathrm{H}_{2} \mathrm{SO}_{4}(98 \%)$, $\mathrm{HCl}(36 \%), \mathrm{H}_{2} \mathrm{O}_{2}(30 \%), \mathrm{KMnO}_{4}, \mathrm{P}_{2} \mathrm{O}_{5}$ and $\mathrm{K}_{2} \mathrm{~S}_{2} \mathrm{O}_{8}$ were purchased from Sinopharm Chemical Reagent Co., Ltd. (Shanghai, China) and used without purification. N, N'-dimethylformamide (DMF) and pyridine were obtained from Xiya Chemical Reagent Company (Chengdu, China) and first refluxed over calcium hydride and then distilled under a reduced pressure prior to use. The epoxy resin used in this study is diglycidylether of bisphenol A (DGEBA, $\mathrm{E}-51$, epoxy value $=0.51$ and viscosity value $=$ $12 \mathrm{~Pa} \cdot \mathrm{s}$ at $25^{\circ} \mathrm{C}$ ) supplied by Yueyang Chemical Plant (Yueyang, China). 4, 4'-diaminodiphenylsulphone (DDS) purchased from Sinopharm Chemical Reagent Co., Ltd. (Shanghai, China), with a molecular mass of 248.31 and purity $>96 \%$ according to the supplier. Deionized (DI) water was used in all the process of aqueous solution preparation and washing.

\subsection{Preparation of graphene oxide (GO)}

The modified Hummers method [24-26] was utilized to oxidize nature flake graphite for the synthesis of GO. The detailed processing is described as following: in a pretreatment step, $\mathrm{K}_{2} \mathrm{~S}_{2} \mathrm{O}_{8}(10 \mathrm{~g})$ and $\mathrm{P}_{2} \mathrm{O}_{5}(10 \mathrm{~g})$ were mixed with $\mathrm{H}_{2} \mathrm{SO}_{4}(98 \%, 80 \mathrm{~mL})$ in a $500 \mathrm{~mL}$ beaker placed in water bath. The solution was stirred and heated to $90^{\circ} \mathrm{C}$ until the reactants were completely dissolved. Graphite powder $(3 \mathrm{~g})$ was then introduced into the acid. The mixture was kept at $90^{\circ} \mathrm{C}$ for $4.5 \mathrm{~h}$ under intensive stirring. Then the mixture was cooled to room temperature and diluted with DI water $(1000 \mathrm{~mL})$ and then filtered and washed to remove the residual acid. The product was dried under ambient conditions overnight.

In the second oxidation step, the pretreated graphite powder was put into $150 \mathrm{~mL} 98 \% \mathrm{H}_{2} \mathrm{SO}_{4}$ at $0^{\circ} \mathrm{C}$. Then $\mathrm{KMnO}_{4}(10 \mathrm{~g})$ was added slowly under stirring, and the temperature of the mixture was kept below $10^{\circ} \mathrm{C}$ by cooling. After that, the mixture was allowed to react at $35 \pm 3^{\circ} \mathrm{C}$ for $2 \mathrm{~h}$. As the reaction progressed, the mixture gradually became pasty. The next step was to cool the suspension temperature to $20^{\circ} \mathrm{C}$, and DI water $(500 \mathrm{~mL})$ was added with vigorous agitation. The mixture was stirred for $6 \mathrm{~h}$, and then additional DI $(700 \mathrm{~mL})$ water was added. $30 \% \mathrm{H}_{2} \mathrm{O}_{2}$ was added to the mixture until the color of the suspension changed to brilliant yellow. For purification, the mixture was rinsed and centrifuged with $5 \% \mathrm{HCl}$ and DI water for several times. The collected graphite oxide was dispersed in DI water by ultrasonication to exfoliate the graphite oxide to $\mathrm{GO}$, the suspension was dried by freeze-drying machine. Finally, GO obtained 1s a spongy solid.

\subsection{Synthesis of the liquid crystalline epoxy grafted graphene oxide (TLCP-g-GO)}

Typical procedure of preparing TLCP-g-GO is described as Figure 1. To functionalize GO, the asprepared GO $(0.1 \mathrm{~g})$ was placed in a $250 \mathrm{~mL}$ dried three-necked flask with $100 \mathrm{~mL}$ DMF/pyridine (10:1) mixing solvent and dispersed through sonication for $30 \mathrm{~min}$. Then $15 \mathrm{~mL}$ DMF containing $28 \mathrm{mmol}$ paraphthaloyl chloride was slowly dropped into the GO suspension at $0 \sim 5^{\circ} \mathrm{C}$, and the mixture was stirred at 

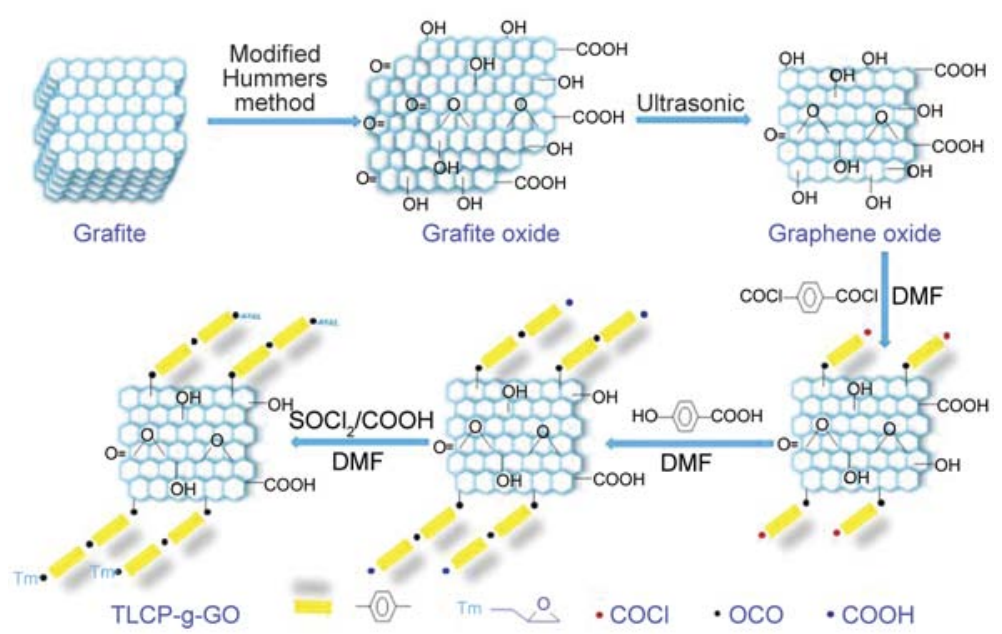

Figure 1. The synthetic routes of TLCP grafted GO

$60^{\circ} \mathrm{C}$ for $12 \mathrm{~h}$. After the reaction finished, $10 \mathrm{~mL}$ $\mathrm{SOCl}_{2}$ was loaded in the flask at ambient temperature. The mixture was kept at $65^{\circ} \mathrm{C}$ under stirring, using a $\mathrm{CaC}_{12}$ guard tube. After $24 \mathrm{~h}$, the excess $\mathrm{SOCl}_{2}$ was removed by distillation, and the product was allowed to react with $5 \mathrm{~mL}$ glycidol at $60^{\circ} \mathrm{C}$ for $12 \mathrm{~h}$. After cooling, the solution was poured into plenty of DI water. The precipitate was collected by filtration and washed with a mixture water/ethanol several times. The solid was vacuum dried at $60^{\circ} \mathrm{C}$ for overnight. The last, TLCP-g-GO was obtained.

\subsection{Preparation of TLCP-g-GO/epoxy composites}

Composition of epoxy composites was as follows. The required amount of filler was first dispersed in acetone by sonication for $30 \mathrm{~min}$. This dispersion was then mixed with epoxy resin to give concentrations of $0.5,1.0,1.5,2.0$ and $3.0 \mathrm{wt} \%$ grafted $\mathrm{GO}$ as compared to the weight of the epoxy resin. The mixture was stirred under high-speed for $0.5 \mathrm{~h}$ for homogenization. In order to evaporate the acetone, the mixture was degassed for at $120^{\circ} \mathrm{C}$ for $2 \mathrm{~h}$. After that, epoxy resin was mixed with stoichiometric amounts of DDS (30 g/100 g of epoxy resin) at $150^{\circ} \mathrm{C}$ for $30 \mathrm{~min}$. The resulting mixture was then cast into a preheated mould coated with silicone resin. The residual solvent and bubbles in the mixture were removed by placing the mold in a vacuum oven for $30 \mathrm{~min}$ at $120^{\circ} \mathrm{C}$. Finally, the mold was placed in a convection oven to cure at $120^{\circ} \mathrm{C}$ for $2 \mathrm{~h}$, $160^{\circ} \mathrm{C}$ for $2 \mathrm{~h}$ and $180^{\circ} \mathrm{C}$ for $2 \mathrm{~h}$. The details of the process of epoxy composites are shown as Figure 2.

\subsection{Characterization}

Fourier-transform infrared spectra (FT-IR) were recorded on a Perkin-Elmer 1710 spectrophotometer. The frequency range of FT-IR was $4000-500 \mathrm{~cm}^{-1}$, using $\mathrm{KBr}$ pellets at room temperature.

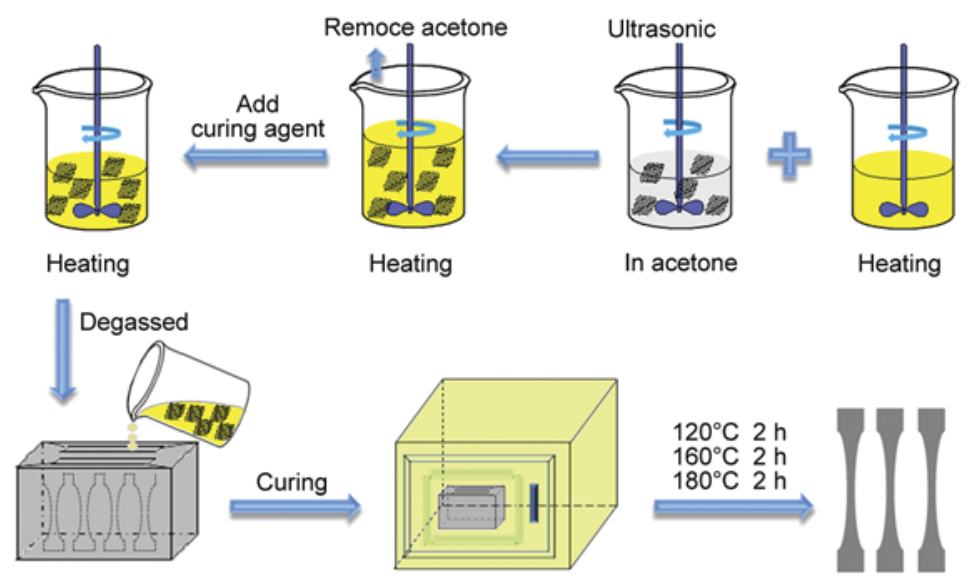

Figure 2. Preparation process of the epoxy composites 
The wide angle X-ray diffraction (WAXD) measurements were carried out on a D8 ADVANCE Xray diffractometer equipped with a computer controller. The scanning was performed from 5 to $50^{\circ}$ with a speed of $2 \% \mathrm{~min}$.

The fracture surfaces of the specimens were investigated using field emission scanning electron microscopy (FE-SEM, JSM-6701F, Japan) at an accelerating voltage of $20 \mathrm{kV}$, and the fracture surfaces were coated with sputter-gold to improve the conductivity.

The liquid crystalline transitions and optical textures were made using a Leica DMxRP polarizing microscope with an INSTEC STC-200 hot stage. Small amounts of samples (2-3 mg) were pre-melted on a microscope slide then covered with a piece of cover glass to form a uniform thin film. The formation and development of the LC phase were examined under polarized light.

The surface functionalization of the GO was analyzed qualitatively by X-ray photoelectron spectroscopy (XPS) using a VG-microtech ESCA2000 spectrometer equipped with a hemispherical electron analyzer and a $\mathrm{MgJa}(\mathrm{hm}=1.2536 \mathrm{keV}) \mathrm{X}$-ray source.

Thermogravimetric analysis (TGA) of the samples with $2 \sim 3 \mathrm{mg}$ was conducted on NETZSCH STA449 from 50 to $700^{\circ} \mathrm{C}$ at a heating rate of $10^{\circ} \mathrm{C} / \mathrm{min}$ under $\mathrm{N}_{2}$.

Differential scanning calorimetry (DSC-204, NET$\mathrm{ZSCH}$, Germany) was performed at temperature from 30 to $250^{\circ} \mathrm{C}$ at heating rate of $10^{\circ} \mathrm{C} / \mathrm{min}$. All tests were performed in a nitrogen atmosphere with a sample weight of about $8 \mathrm{mg}$.

Dynamic mechanical analysis (DMA) was performed on a DMA Q800 dynamic mechanical analyzer (TA Instruments, USA) to determine modulus and glass transition temperature $\left(T_{\mathrm{g}}\right)$. The tests were carried out in the single cantilever mode at a frequency of $1.0 \mathrm{~Hz}$ from 40 to $250^{\circ} \mathrm{C}$ at a heating rate of $3^{\circ} \mathrm{C} / \mathrm{min}$.

Mechanical properties of the composites were evaluated by impact, tensile and flexural measurements. Izod impact strength was measured on a tester of type XJJ-5, which is with no notch in the specimen according to National Standard of China (GB104379). The experiments were carried out on cubic samples $(80 \mathrm{~mm} \times 10 \mathrm{~mm} \times 4 \mathrm{~mm})$. The tensile strength was examined on a universal tensile tester of type RGT-5 according to National Standard of China
(GB1040-92). The tensile rate was $2 \mathrm{~mm} / \mathrm{min}$. Flexural tests were performed according to WDW-20 (Shenzhen Jun Red Instrument Equipment Co., Ltd, China) using a three-point bending mode of the universal testing machine with a crosshead speed of $2 \mathrm{~mm} / \mathrm{min}$. The conditions of the tests and the specimens conformed to GB1449-2005. All the presented results are the average value of five specimens.

\section{Results and discussion}

In order to prove the grafting of TLCP on the surface of GO, FT-IR measurement was conducted. The FT-IR spectra of GO and TLCP-g-GO are presented in Figure 3. GO depicts a strong $\mathrm{OH}$ peak at $3406 \mathrm{~cm}^{-1}[5,24]$. The characteristic adsorption band corresponding to the $\mathrm{C}=\mathrm{O}$ carbonyl stretching is $1732 \mathrm{~cm}^{-1}$, and the $\mathrm{C}-\mathrm{O}$ band is presented at $1053 \mathrm{~cm}^{-1}$. The spectrum also shows a $\mathrm{C}=\mathrm{C}$ peak at $1619 \mathrm{~cm}^{-1}$ corresponding to the remaining sp $\mathrm{spar}^{2}$ ch acter $[27,28]$. Comparing the spectra of TLCP-g-GO with that of GO, several new peaks are observed. Upon grafting, remarkably reduced hydroxyl absorption $\left(3406 \mathrm{~cm}^{-1}\right)$ indicates a shielding effect of TLCP chains, and a sharp absorption peak appears at $1690 \mathrm{~cm}^{-1}$ that can be attributed to the carbonyl stretching vibration of the esters in TLCP. The bands of 1255 and $1070 \mathrm{~cm}^{-1}$ can be explained owing to the $\mathrm{C}-\mathrm{O}-\mathrm{C}$ symmetric and asymmetric stretching vibration. The band of $1160 \mathrm{~cm}^{-1}$ is due to the $\mathrm{C}-\mathrm{O}$ stretching vibration of the ester group in TLCP $[29,30]$. The FT-IR curve affords support for covalent bonding at the interface.

TGA is a complementary technique that can reveal the composition and changes in thermal stability of the samples. As shown in Figure 4a, the samples

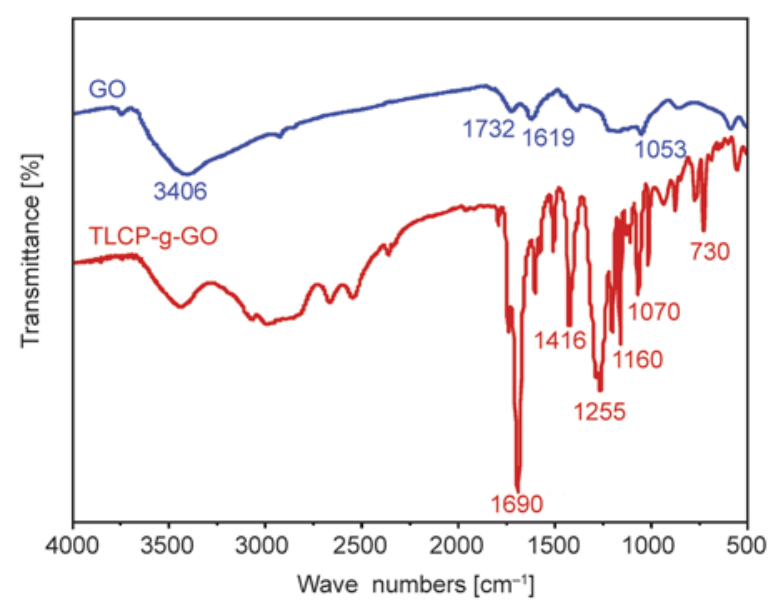

Figure 3. FT-IR spectra of GO and TLCP-g-GO 

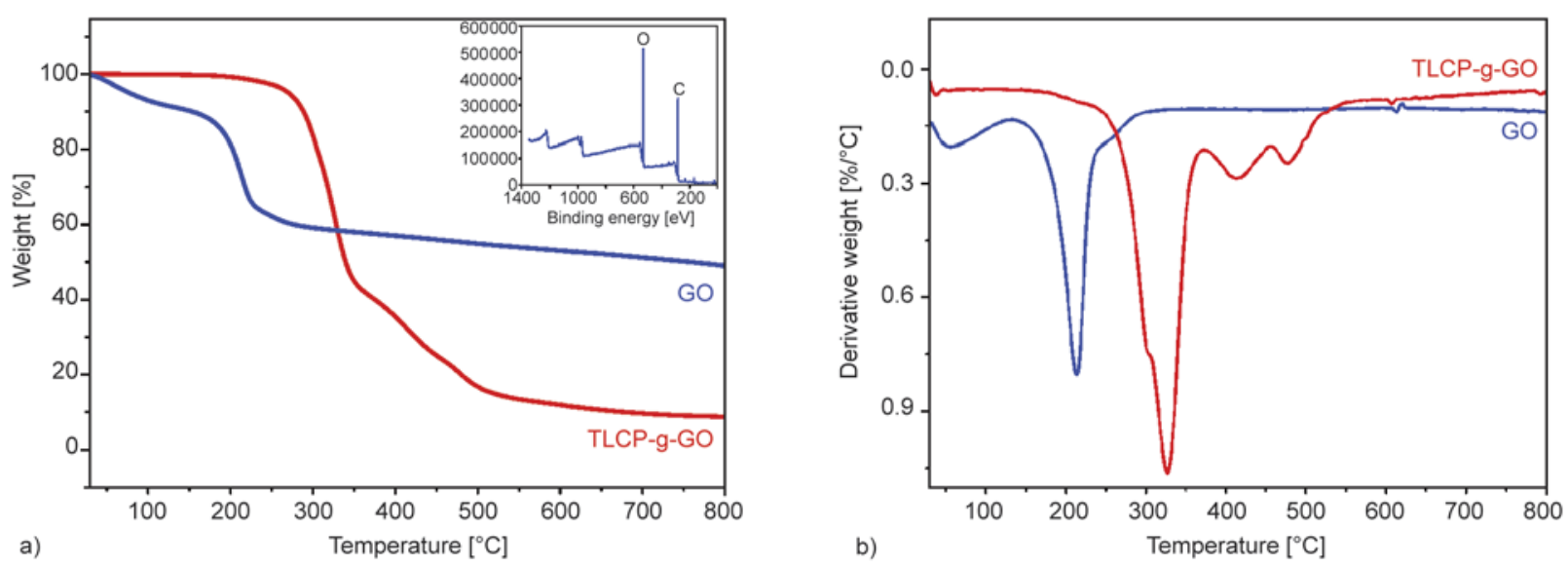

Figure 4. (a) TGA curves of GO and TLCP-g-GO, inset: XPS survey spectra of GO, (b) DTG curves of the GO and TLCP-g-GO

exhibit different thermal behavior. And the XPS survey spectra of GO was inserted in Figure 4a. As shown in the illustration, there are two peaks at 529 and $285 \mathrm{eV}$ corresponding to the $\mathrm{O}_{1 \mathrm{~s}}$ and $\mathrm{C}_{\mathrm{ls}}$ binding energy of $\mathrm{GO}$. The existence of $\mathrm{O}$ element is due to the oxygen-containing functional groups, which is in good agreement with FT-IR analysis. So pristine GO is not thermally stable, and mass loss starts even below $100^{\circ} \mathrm{C}$ and is rapidly at $150^{\circ} \mathrm{C}[13,31]$, which are respectively attributed to evaporation of the remaining water and the decomposition of labile oxygen groups [32]. In contrast, the weight loss of TLCP-g-GO starts at $280^{\circ} \mathrm{C}$, which is roughly $130^{\circ} \mathrm{C}$ higher than that of GO. And the weight retention at $800^{\circ} \mathrm{C}$ for TLCP-g-GO is about $10 \%$. In addition, as can be seen from the DTG curves in Figure 4b, the maximum degradation temperature $\left(T_{\max }\right)$ of the GO also is slightly improved by grafting TLCP. The Tmax is observed at about $213^{\circ} \mathrm{C}$ for $\mathrm{GO}$, and the GO-g-TLCP shifts the corresponding value to about $326^{\circ} \mathrm{C}$, i.e. an evident increase of about $107^{\circ} \mathrm{C}$. All of these indicate that the polymer is indeed attached onto the surface of GO sheets, and the polymer can improve the thermal stability of the GO.

The study by DSC and POM enables us to determine the transition temperatures. The DSC thermograms of TLCP-g-GO are shown in Figure 5. The DSC curves of TLCP-g-GO show a melting transition at $149^{\circ} \mathrm{C}$ and a nematic-isotropic phase transition at $248^{\circ} \mathrm{C}$ on heating, as well as displays an isotropic-nematic phase transition at $237^{\circ} \mathrm{C}$ and a crystallization process at $135^{\circ} \mathrm{C}$ on cooling. The POM results show that TLCP-g-GO exhibits liquid crystalline phase in the heating and cooling cycle. Furthermore, the phase transition temperatures observed by POM are consistent with the DSC

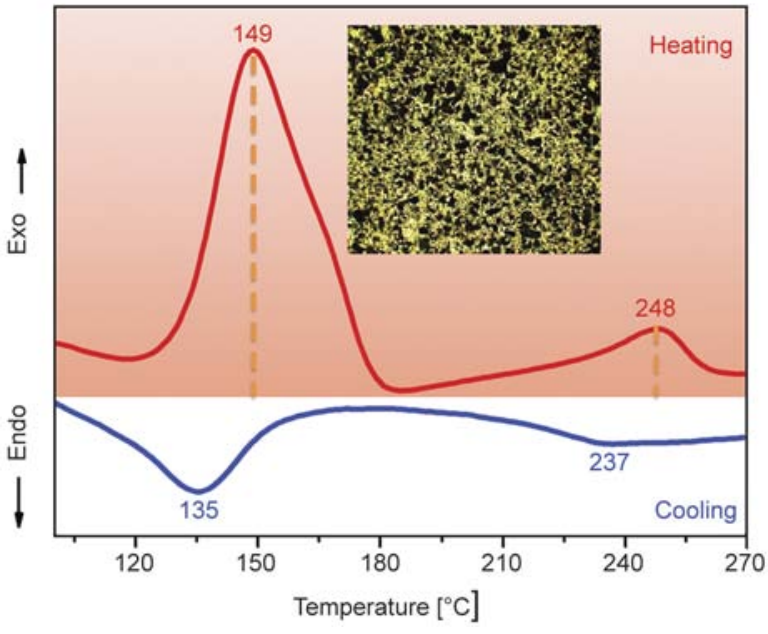

Figure 5. DSC curves of TLCP-g-GO, inset: POM figure of TLCP-g-GO at $200^{\circ} \mathrm{C}$

determination results. The typical optical texture of TLCP-g-GO is shown in inset of Figure 5. It can be seen that the marbled texture of the nematic phase is found to appear between the two peaks in each process.

The wide-angle X-ray scattering patterns of TLCPg-GO and GO are shown in Figure 6. It can be seen that GO spectra shows a strong peak at $9.14^{\circ}$ and a weak, broad peak at $18.60^{\circ}$. However, the strong peak at $9.14^{\circ}$ in the GO pattern is not present in the TLCPg-GO pattern, indicating that TLCP cause the GO sheets to stack more loosely. At the same time, the curve for TLCP-g-GO shows a group of sharp diffraction peaks in the $2 \theta$ region of $15 \sim 30^{\circ}$, but no sharp peaks in the small-angle region appeared, suggesting no smectic layers [33]. All these results indicate that the TLCP-g-GO displays nematic mesophase. Meanwhile, the optical photographs of GO and TLCP-g-GO solution are shown in the inset of Figure 6. GO can be dispersed well in water due to 


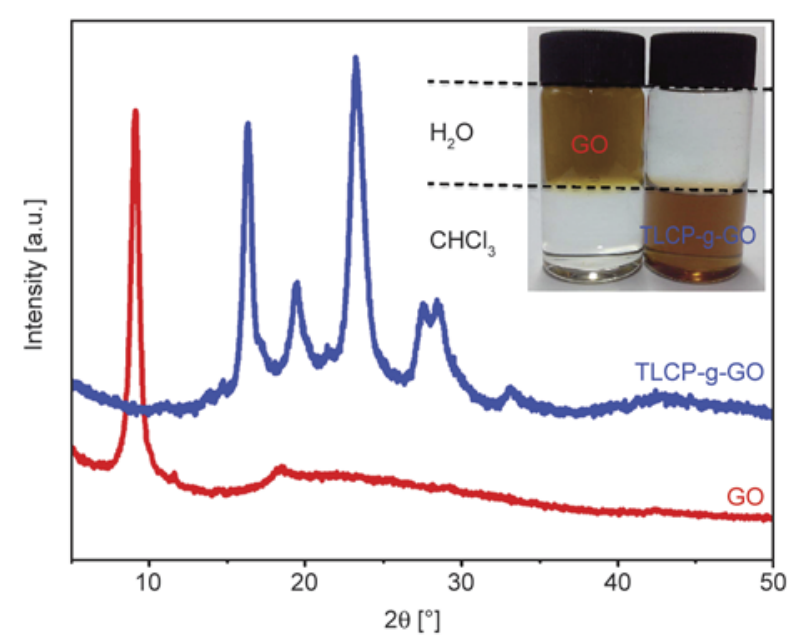

Figure 6. WAXD patterns of GO and TLCP-g-GO, inset: photographs of GO and TLCP-g-GO

the hydroxyl, carboxyl and epoxide groups on its surface. However the TLCP-g-GO only dispersed well in $\mathrm{CHCl}_{3}$ solvent. The optical photographs provide more direct evidence to prove that thermotropic liquid crystalline epoxy is successfully grafted onto the surface of GO.

\subsection{Morphological analysis of the composites}

The morphologies of the fracture surfaces of the neat epoxy and its composites are presented in Figure 7. For each specimen, two types of micrographs are presented, one at a relatively low magnification (Figure 7a, 7c and 7e) and the other at a higher magnification (Figure 7b, 7d and 7f). As shown in Figure $7 a$ and $7 b$, the fracture surface of the neat epoxy exhibits river patterns and very smooth. In addition, the cracks spread freely and randomly, revealing its nature of weak resistance to crack initiation and propagation $[8,34,35]$. Accordingly, the fracture process of neat epoxy is a typical brittle fracture pattern. On the contrary, the composites with TLCP-g-GO exhibit a rougher fracture surface (Figure $7 \mathrm{c}$ and $7 \mathrm{e}$ ), and numerous tortuous and indentations and deep cracks can be observed. The large surface area and liquid crystalline groups significantly improve the interfacial adhesion between the filler and matrix, leading to plastic deformation when impacted. Figure $7 \mathrm{~d}$ reveals that the filler randomly dispersed as a three dimension network through the polymer matrix rather than simply aligning parallel to the surface of the sample. Meanwhile, high embedding and tight binding with epoxy matrix, these further confirm that strong interfacial interaction between filler and epoxy resins. How- ever, with the filler loading increasing, nanosheets exist in the form of agglomerates, as shown in Figure $7 \mathrm{e}$ and $7 \mathrm{f}$. The agglomerates usually delaminate or slip by each other during the fracture process due to the weak adhesion between both the sheet/sheet and sheet/matrix [36].

\subsection{Thermal properties}

Figure 8 shows the change in $T_{\mathrm{g}}$ for the neat epoxy and epoxy composites. Here the intermediate temperature is denoted as the glass transition temperature. It can be observed that the $T_{\mathrm{g}}$ of neat epoxy is $174.9^{\circ} \mathrm{C}$. Meanwhile, the $T_{\mathrm{g}} \mathrm{s}$ shift to higher values with the addition of nanosheets into the epoxy matrix and the $T_{\mathrm{g}}$ of composites increases by $15^{\circ} \mathrm{C}$ when TLCP-g-GO content is $1.5 \mathrm{wt} \%$. This phenomenon is attributed to the following two factors. First, nanosheets confine polymer chains and reduce the chains mobility after introducing nanosheets into the matrix. It is believed that nanosheets can act as physical interlock points in the cured organic matrix, which generally not only provides a sterically hindered environment for curing reactions of composites, but also restrains the chain mobility [37]. Second, the matrix and chemical groups on the filler surface may participate in the curing reaction, leading to the higher crosslinking density.

Figure 9 shows the TGA and DTG curves for all the samples. It is apparently seen that all samples exhibit similar thermal behavior and only one-step decomposition, suggesting that the existence of nanosheets did not significantly alter the degradation mechanism of the epoxy matrix. The composites show a high thermal stability with decomposition temperatures $\left(T_{\mathrm{d}}\right)$ at $5 \%$ weight loss occurred greater than $345^{\circ} \mathrm{C}$. The $T_{\mathrm{d}}$ of epoxy increases from 345.4 to $381.2^{\circ} \mathrm{C}$ by addition of $1.0 \mathrm{wt} \%$ TLCP-gGO. It is also noted the char yields of all composites are increased as compared to neat epoxy. Moreover, as can be seen from the DTG curves, the maximum degradation temperature $\left(T_{\max }\right)$ of the materials also is slightly improved by addition of the nanosheets. The $T_{\max }$ is $18^{\circ} \mathrm{C}$ higher than that of the neat epoxy resin when filler content is $1.0 \mathrm{wt} \%$, and the specific data of thermal decomposition are summarized in Table 1.The significant improvement of thermal stabilities can be attributed to the layered structure of the composite, which causes tortuous pathway for the volatile degradation products, thus delays the decomposition rate of the whole composites 


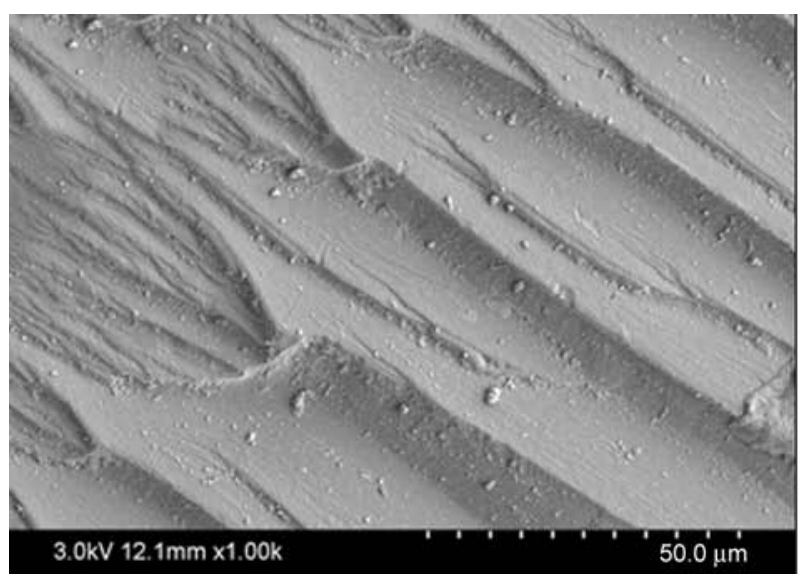

a)

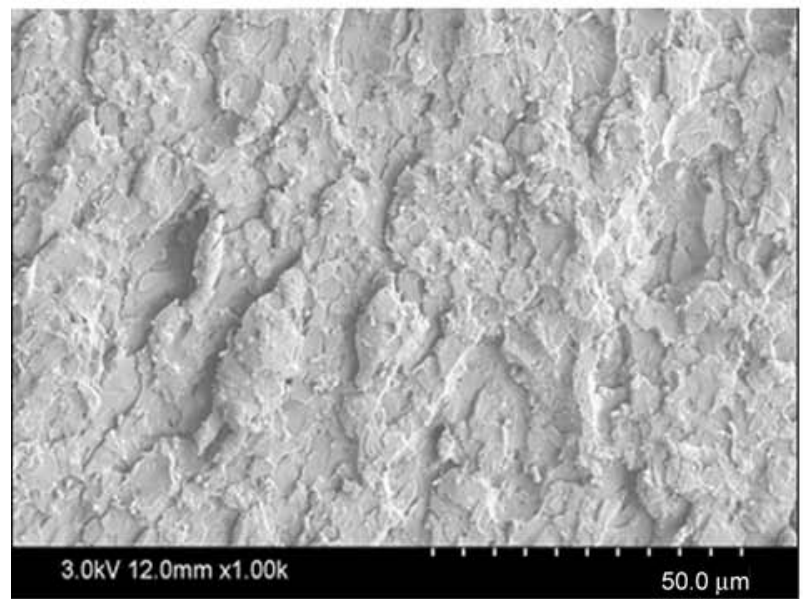

c)

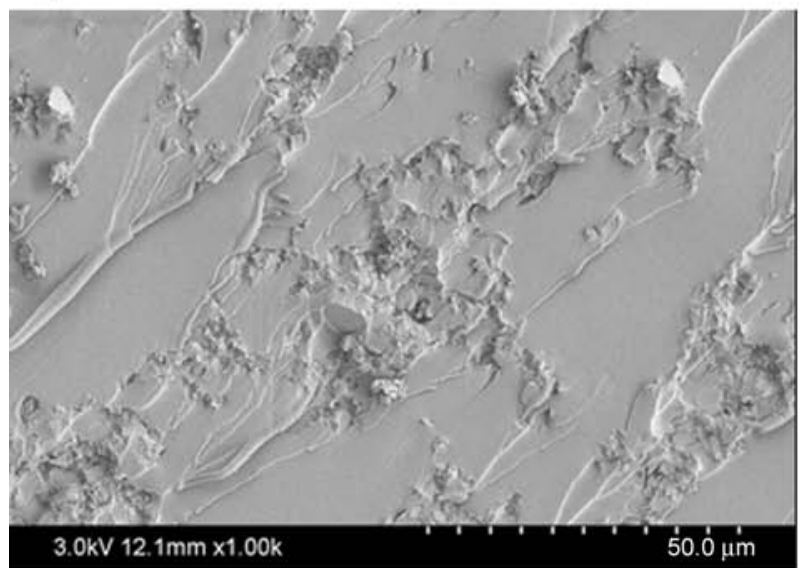

e)

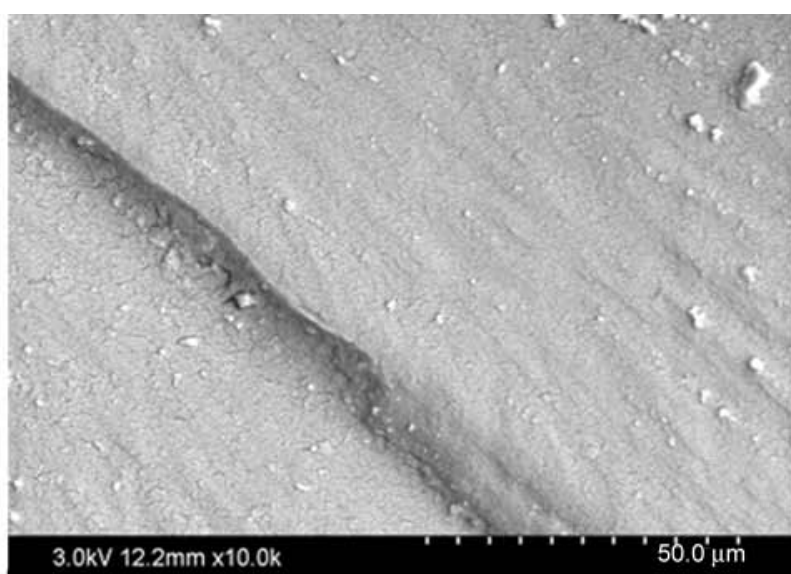

b)

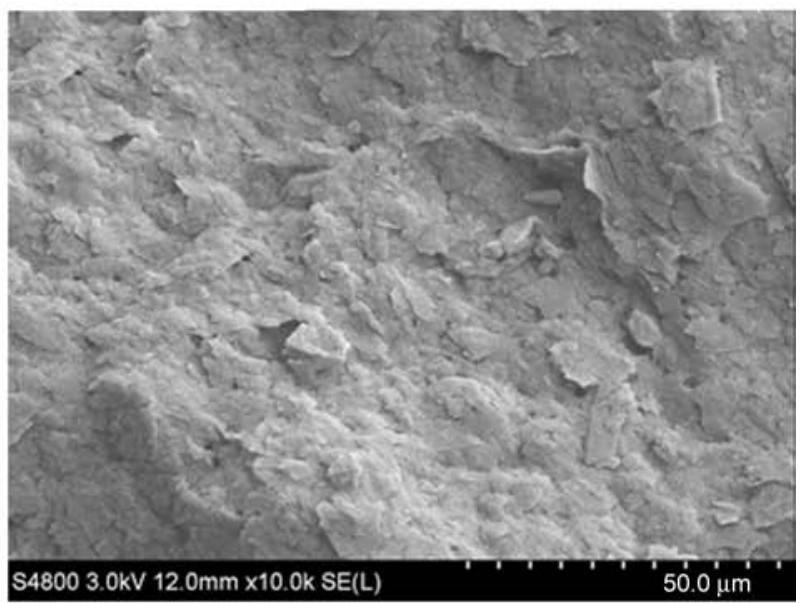

d)

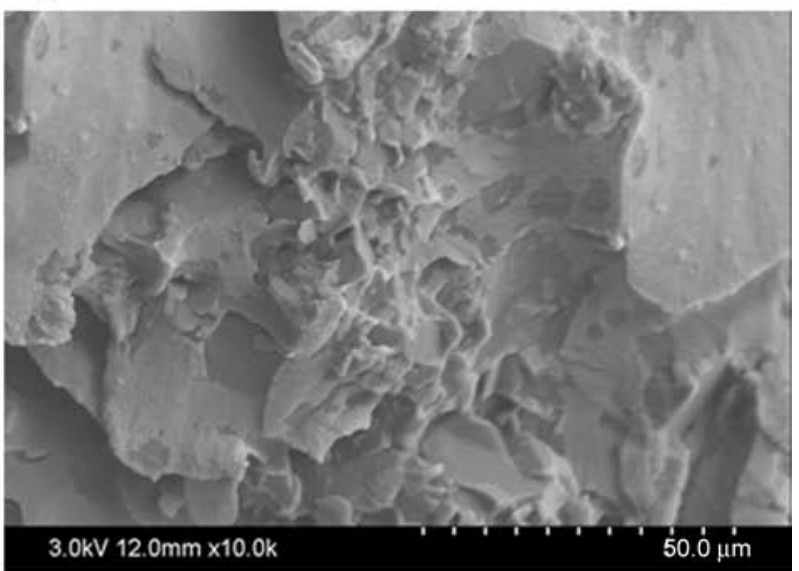

f)

Figure 7. FE-SEM images of fracture surfaces of (a), (b) neat epoxy; (c), (d) $1.5 \mathrm{wt} \%$ epoxy; (e), (f) $3.0 \mathrm{wt} \%$ epoxy

Table 1. The initial decomposition temperatures (IDTs) and maximum degradation temperatures $\left(T_{\max } \mathrm{s}\right)$ of composites

\begin{tabular}{|c|c|c|}
\hline $\begin{array}{c}\text { TLCP-g-GO } \\
\text { content } \\
{[\mathbf{w t} \%]}\end{array}$ & $\begin{array}{c}\text { Initialde composition } \\
\text { temperatures (IDTs) } \\
{\left[{ }^{\circ} \mathbf{C}\right]}\end{array}$ & $\begin{array}{c}\text { Maximum degradation } \\
\text { temperatures }\left(\mathbf{T}_{\max } \mathbf{s}\right) \\
{\left[{ }^{\circ} \mathbf{C}\right]}\end{array}$ \\
\hline 0.0 & 345.4 & 400.1 \\
\hline 0.5 & 371.5 & 407.7 \\
\hline 1.0 & 381.2 & 418.1 \\
\hline 1.5 & 376.9 & 415.5 \\
\hline 2.0 & 376.0 & 412.8 \\
\hline 3.0 & 372.3 & 408.6 \\
\hline
\end{tabular}

$[38,39]$. At the same time, the filler surface and the matrix take place crosslinking by curing reaction, which restrict thermal motion of the polymer molecular and also improve the thermal stability of the polymer.

Figure 10 shows the variations in storage modulus and $\tan \delta$ as a function of temperature from below the glassy state temperature range to the rubbery plateau of neat epoxy and its composites. As shown in Figure 10a, the storage modulus of the materials 


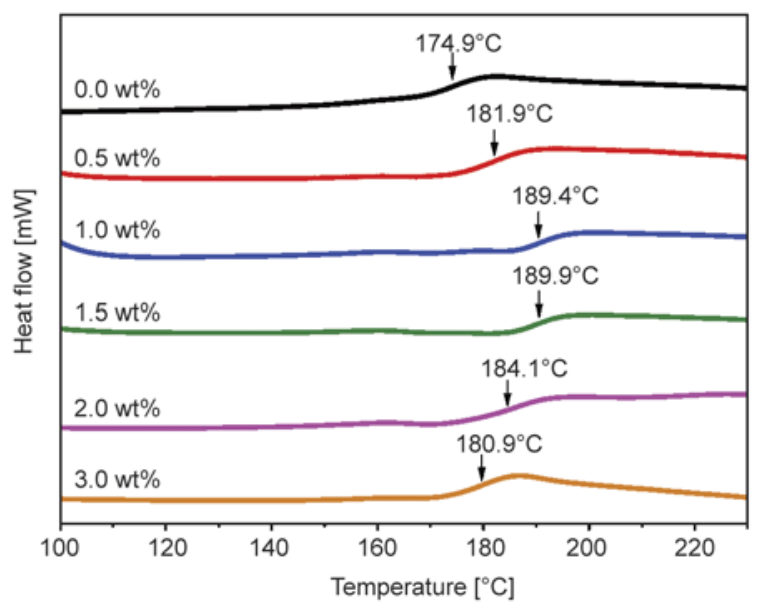

Figure 8. DSC curves of the cured neat epoxy and epoxy composites

was significantly increased by the addition of TLCPg-GO fillers. It can be seen that below $150^{\circ} \mathrm{C}$, the storage modulus of the composites is approximate to the neat epoxy. But in rubbery region, the storage moduli of the composites appears much higher than that of the neat epoxy. For instance, at $165^{\circ} \mathrm{C}$ the storage modulus was about $36 \%$ higher than neat epoxy resin when filler content is $1.5 \mathrm{wt} \%$. This can be

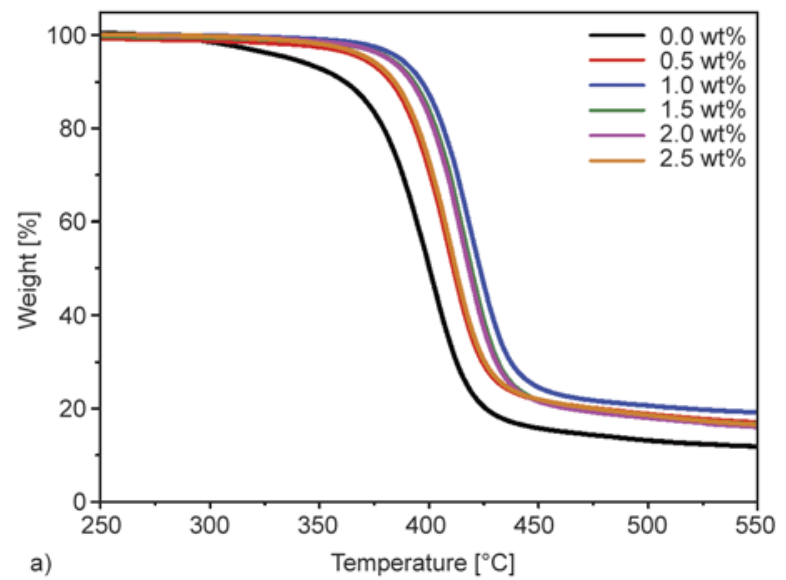

attributed to two reasons. Firstly, the filler effect mentioned earlier, namely filler can act as physical interlock points, which confine polymer chains and reduce the chains mobility [40]. Secondly, the LC domains also act as crosslink, tying segments of the polymer chain together [19]. They do not relax or become soft at higher temperature, and therefore the movements of the polymer chains are restricted by these rigid LC domains. Upon the incorporation of $2.0 \mathrm{wt} \%$ of TLCP-g-GO, however, the storage modulus decreases, probably because the particle loading exceeds the critical level. The excess TLCP$\mathrm{g}$-GO particles form agglomerates in the polymer matrix. The agglomerates affect the homogeneous dispersion of filler in polymer matrix, and reduce the crosslinking of composites.

The $T_{\mathrm{g}}$ measured from the peak of the $\tan \delta$ curve also shows that composites have higher $T_{\mathrm{g}}$ compared to that of neat epoxy. As shown in Figure 10b, the $T_{\mathrm{g}}$ of neat epoxy polymer was about $184.4^{\circ} \mathrm{C}$. With the addition of $1.5 \mathrm{wt} \%$ TLCP-g-GO, the $T_{\mathrm{g}}$ value increased to $198.9^{\circ} \mathrm{C}$. Both of the filler effect and the crosslink effect are responsible for the high

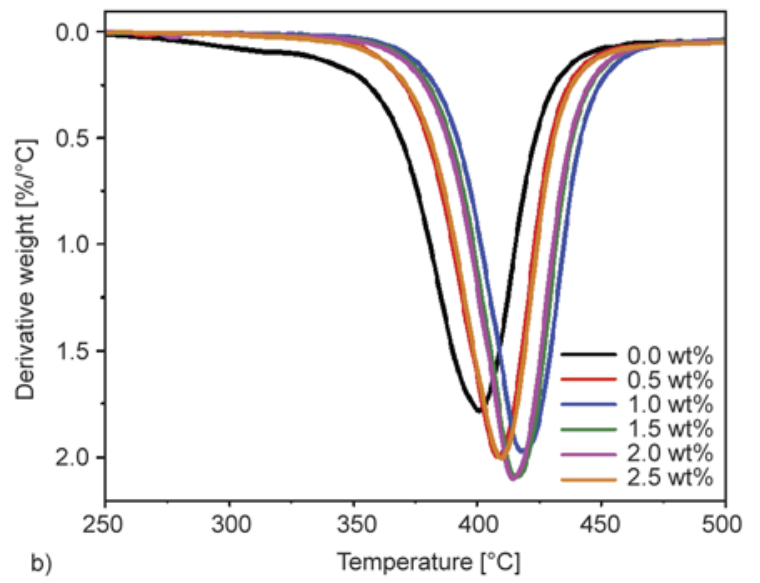

Figure 9. (a) TGA and (b) DTG curves of the neat epoxy and its composites
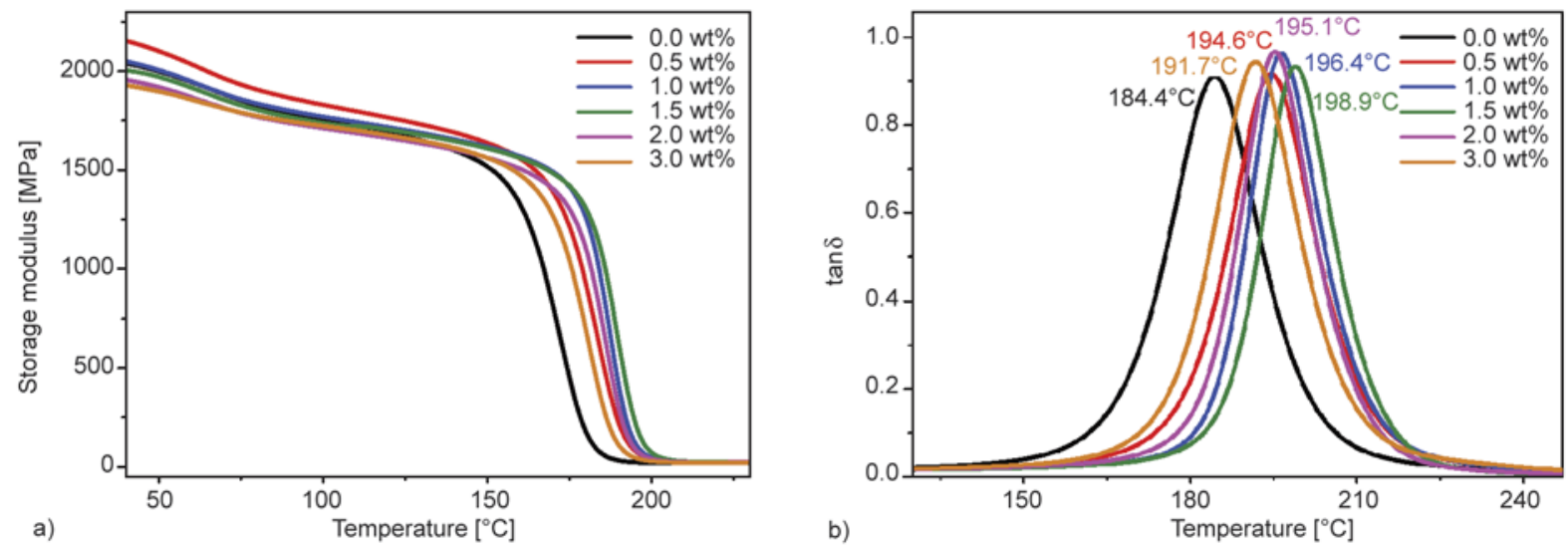

Figure 10. (a) Storage moduli and (b) $\tan \delta$ as a function of temperature of the neat epoxy and its composites 
$T_{\mathrm{g}}$ observed in composites. The free volume of the composites is significantly reduced due to the presence of fillers, thereby decreasing the mobility of the segments in response to an applied thermal energy. While the dramatic drop in the $T_{\mathrm{g}}$ at $2.0 \mathrm{wt} \%$ loading, which can be due to increased agglomeration sites within the composites. The agglomerates are physical imperfections in the epoxy composites, which hinder the curing of resin and lead to the low crosslinking density. The $T_{\mathrm{g}}$ of the polymer was measured using DSC, which is in agreement with the DMA results. Although measured through different experimental technique, the composites always show higher $T_{\mathrm{g}}$ than neat epoxy.

\subsection{Mechanical properties of nanocomposites}

Typical stress-strain curves of the epoxy composites obtained during tensile testing are shown in Figure 11. The tensile strength and elongation at break were varied with the filler contents [41]. As shown in Figure 11, the composites exhibit linear stress-strain character up to failure, without plastic deformation, and every specimen shows a similar curve shape [42]. The results also show an improvement in tensile strength and modulus of composites compared to neat epoxy for all concentrations of the filler. That is to say, the mechanical properties of composites get a high reinforcement by the dispersion of TLCP-g-GO in polymer matrix.

The variations of impact strength and tensile strength according to the filler contents are shown in Figure 12. Obviously, the impact strength and tensile strength of the composites are enhanced effectively with the addition of fillers. As seen in Figure 12, for neat epoxy impact strength and tensile

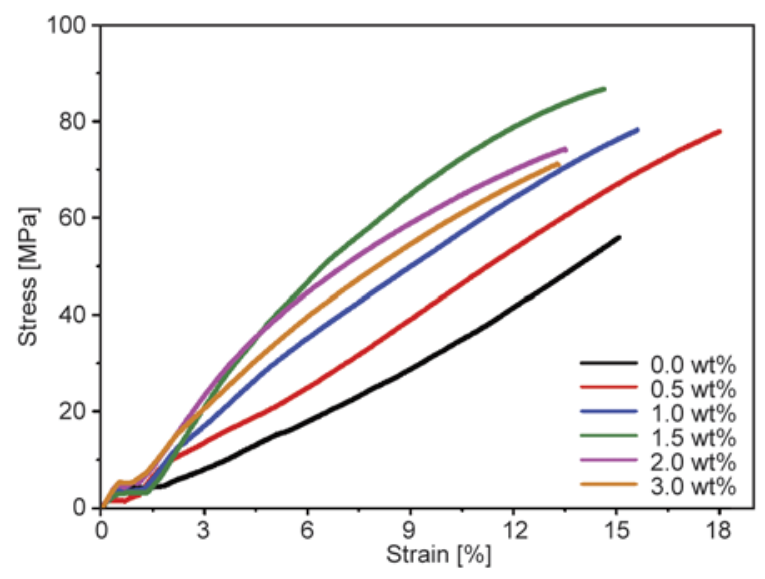

Figure 11. Typical stress-strain curves for the neat epoxy and its composites

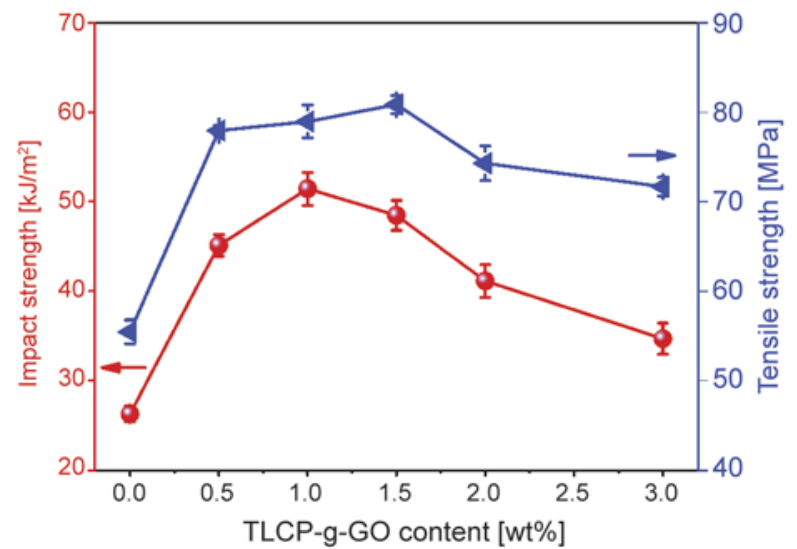

Figure 12. Impact strength and tensile strength of neat epoxy and epoxy composites

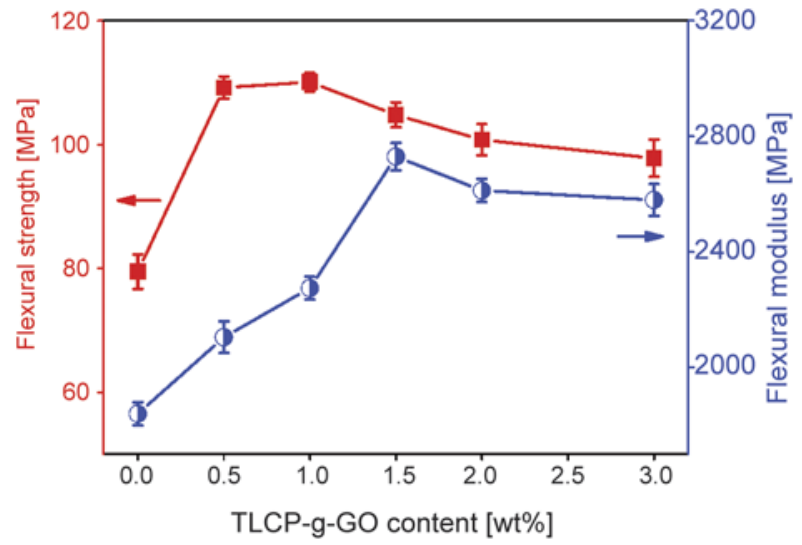

Figure 13. Flexural strength and modulus of neat epoxy and its composites

strength are $26.25 \mathrm{~kJ} / \mathrm{m}^{2}$ and $55.43 \mathrm{MPa}$, respectively. With increasing concentration of TLCP-gGO in the composite, impact strength and tensile strength first increase steeply, reach a plateau and decrease again at higher TLCP-g-GO loadings. The most significant improvement is obtained at a loading with 1.0 and $1.5 \mathrm{wt} \%$ TLCP-g-GO reaching $51.43 \mathrm{~kJ} / \mathrm{m}^{2}$ and $80.85 \mathrm{MPa}$, for impact strength and tensile strength respectively, which are increase of 96 and 46\% compared to neat epoxy. This is attributed to the large surface area and multiple interactions between the functional groups of the filler and matrix in the composites [43, 44].

Figure 13 shows the flexural properties of the neat epoxy and its composites. The results show an improvement in strength and modulus of the composites compared to neat epoxy for all concentrations of TLCP-g-GO. A similar increasing tendency with TLCP-g-GO content is also found for the flexural strength. The composites with the flexural strength values ranging from 97.8 to $110.1 \mathrm{MPa}$, which are much higher than that of neat epoxy 
Table 2. The mechanical properties of neat epoxy and its composites

\begin{tabular}{|c|c|c|c|c|}
\hline $\begin{array}{c}\text { TLCP-g-GO content } \\
{[\mathbf{w t} \% \mathbf{0}]}\end{array}$ & $\begin{array}{c}\text { Impact strength } \\
{\left[\mathbf{k J} / \mathbf{m}^{\mathbf{2}}\right]}\end{array}$ & $\begin{array}{c}\text { Tensile strength } \\
{[\mathbf{M P a}]}\end{array}$ & $\begin{array}{c}\text { Flexural strength } \\
{[\mathbf{M P a}]}\end{array}$ & $\begin{array}{c}\text { Flexural modulus } \\
{[\mathbf{M P a}]}\end{array}$ \\
\hline 0.0 & $26.25 \pm 0.90$ & $55.43 \pm 1.33$ & $79.5 \pm 2.8$ & $1840 \pm 40$ \\
\hline 0.5 & $45.12 \pm 1.21$ & $77.95 \pm 0.55$ & $109.2 \pm 1.8$ & $2105 \pm 55$ \\
\hline 1.0 & $51.43 \pm 1.85$ & $78.96 \pm 0.86$ & $110.1 \pm 1.5$ & $2275 \pm 41$ \\
\hline 1.5 & $48.46 \pm 1.67$ & $80.85 \pm 1.01$ & $104.8 \pm 2.0$ & $2730 \pm 48$ \\
\hline 2.0 & $41.12 \pm 1.82$ & $74.29 \pm 1.95$ & $100.8 \pm 2.5$ & $2613 \pm 40$ \\
\hline 3.0 & $34.67 \pm 1.74$ & $71.76 \pm 1.04$ & $97.8 \pm 3.0$ & $2580 \pm 55$ \\
\hline
\end{tabular}

(79.5 MPa).The maximum improvement in flexural strength is observed at the $1.0 \mathrm{wt} \%$ filler content. The improved flexural strength is attributed to following reasons. Graphene oxide has large surface area, which can serve as connecting bridges to prevent the matrix from fracturing upon mechanical deformation. In addition, liquid crystalline groups have certain rigidity, and it can also improve the properties of epoxy composites. However, as the filler concentration exceeded $1.0 \mathrm{wt} \%$, the flexural strength of the composites decreased suddenly, probably because the particle loading exceeds the critical level, called mechanical percolation [45]. The excess particles may form agglomerates in the polymer matrix, and lead to cracks to initiate and propagate easily, consequently, produce the reduced strength of the composites [36].The modulus of the composites increases steadily with the incorporation of the fillers to $1.5 \mathrm{wt} \%$ of TLCP-g-GO, the maximum improvement is $890 \mathrm{MPa}$. Beyond this concentration, the modulus decreased. Nevertheless, it remained still above that of the neat epoxy. This phenomenon is possibly because the addition of TLCP-g-GO caused a dilution effect in the matrix, i.e. the volume fraction of epoxy resin matrix decreased with increasing filler loading, which would lead to a decrease of crosslinking of composites. In addition, the filler agglomerates in the epoxy composites can delaminate or slip by each other during the external role process due to the weak adhesion between both the sheet/sheet and sheet/ matrix.

In general, a significant improvement in the mechanical properties is attributed to the strong interaction between TLCP-g-GO and epoxy matrix, so that the efficient load transfer between nanosheets and the epoxy matrix [46, 47]. Moreover, the nanosheets may serve as connecting bridges to prevent the matrix from fracturing upon mechanical deformation, thus enhancing the mechanical properties of epoxy. Another reason may be that, during the casting process, the nanosheets are inclined to lay flat and oriented along the flow of epoxy resin direction, which is beneficial for the efficient stress transfer and thus reinforcing the mechanical properties of composites [48, 49]. Table 2 summarizes the mechanical properties data.

\section{Conclusions}

In this paper, we prepared high performance TLCPg-GO/epoxy composites with good thermal properties, high strength and high modulus. By thermotropic liquid crystalline epoxy modified GO ensured excellent dispersity and good compatibility in the polymer matrix. The composites showed a high reinforcing efficiency, which attributed to the special two dimensional morphology of GO and the functionalized surface of GO providing well dispersion, high contact area in matrix. The composites exhibit around a $96 \%$ increase in impact strength, $46 \%$ improvement in tensile strength, and $48 \%$ reinforce in flexural modulus for a low filler content (1.0 or $1.5 \mathrm{wt} \%)$. Meanwhile, with the incorporation of $1.5 \mathrm{wt} \%$ of TLCP-g-GO, the $T_{\mathrm{g}}$ of composites increases by $15^{\circ} \mathrm{C}$. The decomposition temperature $\left(T_{\mathrm{d}}\right)$ at $5 \%$ weight loss is $35.8^{\circ} \mathrm{C}$ higher than that of the neat epoxy resin. In short, we provide a promising approach to prepare graphene based composites with enhanced performances.

\section{Acknowledgements}

The authors gratefully acknowledge the financial support by National Natural Science Foundation of China (51163004 and 51303034), the Natural Science Foundation of Guangxi Province, China (No.2013GXNSFAA019308), the Opening Funding of Guangxi Key Laboratory for Advance Materials and New Preparation Technology (12KF-8), Innovation Team of Guangxi Universities' Talent Highland and Guangxi Funds for Specially-appointed Expert. 


\section{References}

[1] Kim J., Yim B-S., Kim J-M., Kim J.: The effects of functionalized graphene nanosheets on the thermal and mechanical properties of epoxy composites for anisotropic conductive adhesives (ACAs). Microelectronics Reliability, 52, 595-602 (2012). DOI: $10.1016 /$ j.microrel.2011.11.002

[2] Jiao J., Sun X., Pinnavaia T. J.: Mesostructured silica for the reinforcement and toughening of rubbery and glassy epoxy polymers. Polymer, 50, 983-989 (2009). DOI: $10.1016 /$ j.polymer.2008.12.042

[3] Shen X-J., Pei X-Q., Fu S-Y., Friedrich K.: Significantly modified tribological performance of epoxy nanocomposites at very low graphene oxide content. Polymer, 54, 1234-1242 (2013).

DOI: 10.1016/j.polymer.2012.12.064

[4] Shen X-J., Pei X-Q., Liu Y., Fu S-Y.: Tribological performance of carbon nanotube-graphene oxide hybrid/ epoxy composites. Composites Part B: Engineering, 57, 120-125 (2014).

DOI: 10.1016/j.compositesb.2013.09.050

[5] Jiang T., Kuila T., Kim N. H., Ku B-C., Lee J. H.: Enhanced mechanical properties of silanized silica nanoparticle attached graphene oxide/epoxy composites. Composites Science and Technology, 79, 115-125 (2013).

DOI: $10.1016 /$ j.compscitech.2013.02.018

[6] Deng S. H., Zhou X. D., Zhu M. Q., Fan C. J., Lin Q. F.: Interfacial toughening and consequent improvement in fracture toughness of carbon fiber reinforced epoxy resin composites: Induced by diblock copolymers. Express Polymer Letters, 7, 925-935 (2013).

DOI: $10.3144 /$ expresspolymlett.2013.90

[7] Chen J., Taylor A. C.: Epoxy modified with triblock copolymers: Morphology, mechanical properties and fracture mechanisms. Journal of Materials Science, 47, 4546-4560 (2012).

DOI: $10.1007 / \mathrm{s} 10853-012-6313-6$

[8] Tang L-C., Zhang H., Sprenger S., Ye L., Zhang Z.: Fracture mechanisms of epoxy-based ternary composites filled with rigid-soft particles. Composites Science and Technology, 72, 558-565 (2012).

DOI: $10.1016 /$ j.compscitech.2011.12.015

[9] Lu S-R., Jiang Y-M., Wei C.: Preparation and characterization of $\mathrm{EP} / \mathrm{SiO}_{2}$ hybrid materials containing PEG flexible chain. Journal of Materials Science, 44, $4047-$ 4055 (2009).

DOI: $10.1007 / \mathrm{s} 10853-009-3584-7$

[10] Johnsen B. B., Kinloch A. J., Mohammed R. D., Taylor A. C., Sprenger S.: Toughening mechanisms of nanoparticle-modified epoxy polymers. Polymer, 48, 530 541 (2007).

DOI: $10.1016 /$ j.polymer.2006.11.038
[11] Shen X-J., Liu Y., Xiao H-M., Feng Q-P., Yu Z-Z., Fu S-Y.: The reinforcing effect of graphene nanosheets on the cryogenic mechanical properties of epoxy resins. Composites Science and Technology, 72, 1581-1587 (2012). DOI: 10.1016/j.compscitech.2012.06.021

[12] Hou S., Su S., Kasner M. L., Shah P., Patel K., Madarang C. J.: Formation of highly stable dispersions of silane-functionalized reduced graphene oxide. Chemical Physics Letters, 501, 68-74 (2010).

DOI: $10.1016 /$ j.cplett.2010.10.051

[13] Yang Y., Wang J., Zhang J., Liu J., Yang X., Zhao H.: Exfoliated graphite oxide decorated by PDMAEMA chains and polymer particles. Langmuir, 25, 1180811814 (2009).

DOI: $10.1021 / 1 a 901441 p$

[14] Shen J., Hu Y., Li C., Qin C., Ye M.: Synthesis of amphiphilic graphene nanoplatelets. Small, 5, 82-85 (2009).

DOI: $10.1002 / \mathrm{smll} .200800988$

[15] Chen G., Zhai S., Zhai Y., Zhang K., Yue Q., Wang L., Zhao J., Wang H., Liu J., Jia J.: Preparation of sulfonic-functionalized graphene oxide as ion-exchange material and its application into electrochemiluminescence analysis. Biosensors and Bioelectronics, 26, 3136-3141 (2011).

DOI: $10.1016 /$ j.bios.2010.12.015

[16] Meng F-B., Cui Y., Chen H-B., Zhang B-Y., Jia C.: Phase behaviors of comb-like liquid crystalline polysiloxanes containing fluorinated mesogenic units. Polymer, 50, 1187-1196 (2009).

DOI: 10.1016/j.polymer.2009.01.005

[17] Jannesari A., Ghaffarian S. R., Mohammadi N., Taromi F. A., Molaei A.: Liquid crystalline thermosets as binder for powder coatings: Thermoanalytical study of the cure characteristics of a carboxylated main chain liquid crystalline oligoester. Progress in Organic Coatings, 50, 213-223 (2004).

DOI: $10.1016 /$ j.porgcoat.2004.02.006

[18] Mija A., Navard P., Peiti C., Babor D., Guigo N.: Shear induced structuration of liquid crystalline epoxy thermosets. European Polymer Journal, 46, 1380-1387 (2010).

DOI: $10.1016 /$ j.eurpolymj.2010.04.001

[19] Li Y., Badrinarayanan P., Kessler M. R.: Liquid crystalline epoxy resin based on biphenyl mesogen: Thermal characterization. Polymer, 54, 3017-3025 (2013). DOI: $10.1016 /$ j.polymer.2013.03.043

[20] Mormann W., Bröcher M.: Liquid crystalline thermosets by polymerization of mesogenic azomethine based diepoxides-influence of reaction rate on phase behaviour. Polymer, 40, 193-198 (1999). DOI: $10.1016 / \mathrm{s} 0032-3861(98) 00211-0$ 
[21] Jaisankar S. N., Nelson D. J., Brammer C. N.: New synthesis and characterization of ionic polyurethane-urea liquid crystals. Polymer, 50, 4775-4780 (2009).

DOI: $10.1016 /$ j.polymer.2009.07.049

[22] Hsu S-H., Wu M-C., Chen S., Chuang C-M., Lin S-H., Su W-F.: Synthesis, morphology and physical properties of multi-walled carbon nanotube/biphenyl liquid crystalline epoxy composites. Carbon, 50, 896-905 (2012).

DOI: 10.1016/j.carbon.2011.09.051

[23] Iqbal M., Dingemans J. T.: High $T_{\mathrm{g}}$ nematic thermosets: Synthesis, characterization and thermo-mechanical properties. European Polymer Journal, 46, 2174 2180 (2010).

DOI: $10.1016 /$ j.eurpolymj.2010.08.010

[24] Ren L., Wang X., Guo S., Liu T.: Functionalization of thermally reduced graphene by in situ atom transfer radical polymerization. Journal of Nanoparticle Research, 13, 6389-6396 (2011).

DOI: 10.1007/s11051-011-0391-2

[25] Guo J., Zhu S., Chen Z., Li Y., Yu Z., Liu Q., Li J., Feng C., Zhang D.: Sonochemical synthesis of $\mathrm{TiO}_{2}$ nanoparticles on graphene for use as photocatalyst. Ultrasonics Sonochemistry, 18, 1082-1090 (2011).

DOI: 10.1016/j.ultsonch.2011.03.021

[26] Akhavan O.: The effect of heat treatment on formation of graphene thin films from graphene oxide nanosheets. Carbon, 48, 509-519 (2010).

DOI: $10.1016 /$ j.carbon.2009.09.069

[27] Song S. H., Park K. H., Kim B. H., Choi Y. W., Jun G. H., Lee D. J., Kong B-S., Paik K-W., Jeon S.: Enhanced thermal conductivity of epoxy-graphene composites by using non-oxidized graphene flakes with non-covalent functionalization. Advanced Materials, 25, 732737 (2013).

DOI: $10.1002 /$ adma.201202736

[28] Gudarzi M. M., Sharif F.: Enhancement of dispersion and bonding of graphene-polymer through wet transfer of functionalized graphene oxide. Express Polymer Letters, 6, 1017-1031 (2012).

DOI: 10.3144/expresspolymlett.2012.107

[29] Wang J-W., Zhang B-Y.: Effect of fluorinated nematic mesogens on phase behaviors and optical properties of chiral liquid crystalline polysiloxanes. Colloid and Polymer Science, 291, 2917-2925 (2013).

DOI: $10.1007 / \mathrm{s} 00396-013-3049-\mathrm{Z}$

[30] Meng F-B., Zhou N-Y., Du C., Gao H-M., He X-Z.: Synthesis, characterization and electrorheological effect of sulfosalt-type liquid-crystalline ionomers containing polyaniline units. Journal of Applied Polymer Science, 130, 3395-3403 (2013).

DOI: $10.1002 / a p p .39593$

[31] Qiu S. L., Wang C. S., Wang Y. T., Liu C. G., Chen X. Y., Xie H. F., Huang Y. A., Cheng R. S.: Effects of graphene oxides on the cure behaviors of a tetrafunctional epoxy resin. Express Polymer Letters, 5, 809818 (2011).

DOI: $10.3144 /$ expresspolymlett.2011.79
[32] Fang M., Wang K., Lu H., Yang Y., Nutt S.: Covalent polymer functionalization of graphene nanosheets and mechanical properties of composites. Journal of Materials Chemistry, 19, 7098-7105 (2009).

DOI: $10.1039 / \mathrm{b} 908220 \mathrm{~d}$

[33] Liu J-H., Hsieh F-M.: Synthesis and characterization of novel liquid crystalline polymers containing cholesteryl pendant groups. Materials Chemistry and Physics, 118, 506-512 (2009).

DOI: $10.1016 / \mathrm{j}$. matchemphys.2009.08.031

[34] Ai L., Zhang C., Chen Z.: Removal of methylene blue from aqueous solution by a solvothermal-synthesized graphene/magnetite composite. Journal of Hazardous Materials, 192, 1515-1524 (2011).

DOI: $10.1016 /$ j.jhazmat.2011.06.068

[35] Wang S., Tambraparni M., Qiu J., Tipton J., Dean D.: Thermal expansion of graphene composites. Macromolecules, 42, 5251-5255 (2009).

DOI: $10.1021 / \mathrm{ma} 900631 \mathrm{c}$

[36] Tang L-C., Wan Y-J., Yan D., Pei Y-B., Zhao L., Li YB., Wu L-B., Jiang J-X., Lai G-Q.: The effect of graphene dispersion on the mechanical properties of graphene/epoxy composites. Carbon, 60, 16-27 (2013). DOI: $10.1016 /$ j.carbon.2013.03.050

[37] Yu J., Huang X., Wu C., Wu X., Wang G., Jiang P.: Interfacial modification of boron nitride nanoplatelets for epoxy composites with improved thermal properties. Polymer, 53, 471-480 (2012).

DOI: $10.1016 /$ j.polymer.2011.12.040

[38] Wu X., Wang Y., Xie L., Yu J., Liu F., Jiang P.: Thermal and electrical properties of epoxy composites at high alumina loadings and various temperatures. Iranian Polymer Journal, 22, 61-73 (2013). DOI: $10.1007 / \mathrm{s} 13726-012-0104-4$

[39] Lu S., Li S., Yu J., Yuan Z., Qi B.: Epoxy nanocomposites filled with thermotropic liquid crystalline epoxy grafted graphene oxide. RSC Advances, 3, 8915-8923 (2013). DOI: $10.1039 / \mathrm{c} 3 \mathrm{ra} 40404 \mathrm{~h}$

[40] Sridhar V., Lee I., Chun H. H., Park H.: Graphene reinforced biodegradable poly(3-hydroxybutyrate-co-4hydroxybutyrate) nano-composites. Express Polymer Letters, 7, 320-328 (2013). DOI: $10.3144 /$ expresspolymlett.2013.29

[41] Feng Q-P., Shen X-J., Yang J-P., Fu S-Y., Mai Y-W., Friedrich K.: Synthesis of epoxy composites with high carbon nanotube loading and effects of tubular and wavy morphology on composite strength and modulus. Polymer, 52, 6037-6045 (2011).

DOI: $10.1016 /$ j.polymer.2011.10.049

[42] Yuan X. Y., Zou L. L., Liao C. C., Dai J. W.: Improved properties of chemically modified graphene/poly(methyl methacrylate) nanocomposites via a facile in-situ bulk polymerization. Express Polymer Letters, 6, 847-858 (2012).

DOI: $\underline{10.3144 / \text { expresspolymlett.2012.90 }}$ 
[43] Zhao Y., Chen Z-K., Liu Y., Xiao H-M., Feng Q-P., Fu S-Y.: Simultaneously enhanced cryogenic tensile strength and fracture toughness of epoxy resins by carboxylic nitrile-butadiene nano-rubber. Composites Part A: Applied Science and Manufacturing, 55, 178-187 (2013).

DOI: $10.1016 /$ j.compositesa.2013.09.005

[44] Chatterjee S., Wang J. W., Kuo W. S., Tai N. H., Salzmann C., Li W. L., Hollertz R., Nüesch F. A., Chu B. T. T.: Mechanical reinforcement and thermal conductivity in expanded graphene nanoplatelets reinforced epoxy composites. Chemical Physics Letters, 531, 610 (2012).

DOI: $10.1016 /$ j.cplett.2012.02.006

[45] Dong J., Yin C., Zhao X., Li Y., Zhang Q.: High strength polyimide fibers with functionalized graphene. Polymer, 54, 6415-6424 (2013). DOI: $10.1016 /$ j.polymer.2013.09.035
[46] Li Y., Umer R., Samad Y. A., Zheng L., Liao K.: The effect of the ultrasonication pre-treatment of graphene oxide (GO) on the mechanical properties of GO/polyvinyl alcohol composites. Carbon, 55, 321-327 (2013). DOI: $10.1016 /$ j.carbon.2012.12.071

[47] Li Y., Pan D., Chen S., Wang Q., Pan G., Wang T.: In situ polymerization and mechanical, thermal properties of polyurethane/graphene oxide/epoxy nanocomposites. Materials and Design, 47, 850-856 (2013).

DOI: $10.1016 /$ j.matdes.2012.12.077

[48] Lv S. H., Ma Y. J., Qiu C. C., Sun T., Liu J. J., Zhou Q. F.: Effect of graphene oxide nanosheets of microstructure and mechanical properties of cement composites. Construction and Building Materials, 49, 121-127 (2013).

DOI: 10.1016/j.conbuildmat.2013.08.022

[49] Satti A., Larpent P., Gun'ko Y.: Improvement of mechanical properties of graphene oxide/poly(allylamine) composites by chemical crosslinking. Carbon, $\mathbf{4 8}$, 3376-3381 (2010).

DOI: $\underline{10.1016 / \mathrm{j} . \text { carbon.2010.05.030 }}$ 